\title{
On the Performance of Garch Family Models in the Presence of Additive Outliers
}

\author{
Emenogu, G. Ngozi,$^{1 *}$ Adenomon, M. O..$^{2^{*}}$ and Nweze, N. O. ${ }^{2}$ \\ 1. Department of Statistics, Federal Polytechnic, Bida, Niger State, Nigeria \\ 2. Department of Statistics, Nasarawa State University, Keffi, Nasarawa State, Nigeria \\ * Corresponding Author: adenomonmo@nsuk.edu.ng
}

$+2348036759112$

\begin{abstract}
It is a common practice to detect outliers in a financial time series in order to avoid the adverse effect of additive outliers. This paper investigated the performance of GARCH family models (sGARCH; gjrGARCH; iGARCH; TGARCH and NGARCH) in the presence of different sizes of outliers (small, medium and large) for different time series lengths (250, 500, 750, 1000, 1250 and 1500) using root mean square error (RMSE) and mean absolute error (MAE) to adjudge the models. In a simulation iteration of 1000 times in $\mathrm{R}$ environment using rugarch package, results revealed that for small size of outliers, irrespective of the length of time series, iGARCH dominated, for medium size of outliers, it was sGARCH and gjrGARCH that dominated irrespective of time series length, while for large size of outliers, irrespective of time series length, gjrGARCH dominated. The study further leveled that in the presence of additive outliers on time series analysis, both RMSE and MAE increased as the time series length increased.
\end{abstract}

Key words: Additive Outliers, Models, Simulation, Time Series length, R Software

\subsection{Introduction}

Response variables are not only affected by exogenous variables but also by themselves from their past behavior. On the basis of this theoretical underpinning, autoregressive models have been invented. Box and Jenkins time series modeling is indispensable in analyzing stochastic processes. Autoregressive and moving average models are used frequently by many disciplines. The autoregressive framework has very useful application in macroeconomics, such as for money supply, interest rate, price, inflation, exchange rates and gross domestic product and in financial time series analysis. The autoregressive heteroskedastic modeling framework is used in 
financial economics, such as asset pricing, portfolio selection, option pricing, and hedging and risk management (Ali, 2013). Studies abound in the financial literature about modeling the return on stocks. Usually, in the financial market, upward movements in stock prices are followed by lower volatilities, while negative movements of the same magnitude are followed by much higher volatilities (Ali, 2013).

Engle (1982) developed the time varying variance model known as autoregressive conditional heteroskedastcity $(\mathrm{ARCH})$ model which was the first model to assume that the volatility is not constant. Bollerslev (1986) extended the model to include the ARMA structure as generalized autoregressive conditional heteroskedasticity (GARCH). Ali (2013) asserted that subsequently, a number of studies have adopted the autoregressive conditional heteroskedasticity (ARCH) or a generalized autoregressive conditional heteroskedasticity (GARCH) models to explain volatility of the stock market; some of these studies have also transformed and developed Engel's basic model to more sophisticated models, such as generalized autoregressive conditional heteroskedasticity (GARCH) model, integrated GARCH (IGARCH), threshold GARCH (TGARCH), exponential GARCH (EGARCH) models, GARCH-in mean (GARCH-M) and others (Atoi, 2014; Grek, 2014); however these sophisticated models, in most case, failed to make the forecast accuracy of the original ARCH model better.

\subsection{Outliers}

In Statistics, an outlier is an observation point that is distant from the other observations. An outlier may be due to variability in the measurement or it may indicate experimental error; the later are sometimes excluded from the data set. An outlier can cause serious problems in statistical analyses. Outliers can occur by chance in any distribution, but they often indicate either measurement error or that the population has a heavy-tailed distribution. In the former case 
one wishes to discard them or use statistics that are robust to outliers, while in the later case they indicate that the distribution has high skewness and that one should be very cautious in using tools or intuitions that assume a normal distribution (Wikipedia, 2017).

There are two types of outliers, namely: innovation outlier (IO), in which an outlier affects future values of the series, and additive outlier (AO), in which an outlier affects only the current observation (McQuarrie \& Tsai, 2003). It should be noted that additive outliers affects forecast performance of GARCH models such that the sum of squares increases as additive outlier increases to a large number,(McQuarrie \& Tsai, 2003).

This study focuses on the impact of additive outliers on performance of GARCH family models. Consequently, some GARCH models are reviewed and the impacts of additive outliers on the GARCH models are examined. Furthermore the study carried out simulation of the GARCH family models in the presence of outliers, assuming three levels of outliers (small, medium and large) at different time series length. The simulation is replicated 1,000 times for each level of outliers and at different time series length, and the performance of the GARCH models is judged using the mean absolute error (MAE) and the root mean square error (RMSE).

\subsection{Justification}

There is need to have appropriate forecasting models which seek to improve forecast performance in financial time series, especially when there are additive outliers, which sort of violates some assumptions of the model.

Since additive outliers affect forecast performance of GARCH models such that the sum of squares increases as additive outlier increases to a large number, this study will reveal the GARCH model(s) which are more robust in forecasting volatility when additive outliers exist. 
The aim of this study is to compare the family of GARCH models when the problem of outliers exists in a financial time series.

\subsection{Literature Review}

\subsection{The GARCH Family Models}

The autoregressive conditional heteroskedasticity $(\mathrm{ARCH})$ model introduced by Fredrick Engel in 1982 is the first model that assumed that volatility is not constant. ARCH models are commonly employed in modelling financial time series that exhibit time-varying volatility clustering, that is, period of swings interspersed with periods of relative calm. (Grek, 2014; Wikipedia, 2017).

Over the years the ARCH model has seen several modifications and extensions resulting in different forms of the generalized autoregressive conditional heteroskedasticity (GARCH) models. GARCH model, which is an extension of ARCH model with autoregressive moving average (ARMA) formulation, was proposed independently by Bollerslev (1986) and Tylor (1986) in order to model in a parsimonious way, and to solve some discovered disadvantages of ARCH model, this position was collaborated by Rossi (2004), Ragnarsson (2011) and Kelkay \& G/Yohannes (2014).

Exponential generalized autoregressive conditional heteroskedasticity (EGARCH) model proposed by Nelson (1991) to overcome some weaknesses of the GARCH model in handling financial time series. In particular, to allow for asymmetric effects between positive and negative asset returns. The log of the conditional variance in EGARCH signifies that the leverage effect is exponential and not quadratic. And (Tsay, 2005; Majose, 2010 and Atoi, 2014) assert that the 
transformation of volatility by its logarithm removes the restriction on the parameter to guarantee the positivity of the variance.

The Nonlinear Generalized Autoregressive Conditional Heteroskedasticity (NGARCH) Model which Higgins \& Bera (1992), Hsieh \& Ritchken (2005) and Duan, et al (2006) said is an important modification of the GARCH model as it exhibits the leverage effect, a very attractive feature of stock return data, by shifting the minimum of the news impact curve away from the origin.

Other extensions of the model include the Glosten-Jagannathan-Runkle GARCH (GJR-GARCH) model proposed by Glosten, et al (1993) models asymmetry in the ARCH process. The model assumes a specific parametric form for the conditional heteroskedasticity present in zero mean white noise series which, although being serially uncorrelated, does not need to be serially independent. The Threshold GARCH (TGARCH) model by Zakoian (1994) is similar to GJR GARCH. It is commonly used to handle leverage effects. It allows the conditional standard deviation to depend on the sign of lagged innovation, and it does not show parameter restriction to guarantee that the conditional variance to be positive. The Integrated Generalized Autoregressive Conditional Heteroskedasticity (IGARCH) model is a restricted version of the GARCH model, where the persistent parameters sum up to one, and imports a unit root in the GARCH process. The Quadratic Generalized Autoregressive Conditional Heteroskedasticity (QGARCH) model by Sentana (1995) is also used to model asymmetric effects of positive and negative shocks. Hentschel (1995) proposed the family GARCH (fGARCH) model as an omnibus model that nests a variety of other popular symmetric and asymmetric GARCH models including APARCH, GJR, AVGARCH, NGARCH, and so on. And the Skew-Generalized Autoregressive Conditional Heteroskedasticity (SGARCH) Model was introduced by De Luca, 
Genton and Loperfido (2005). It is a GARCH structure that takes into account the heteroskedastic nature of financial time series. It allows for parsimonious modeling of multivariate skewness, and according to De Luca and Loperfido (2012), all its elements are either null or negative, consistently with previous empirical and theoretical findings.

\subsection{Methodology}

This study focuses on the GARCH models that are robust for forecasting the volatility of financial time series data in the presence of outliers; so GARCH model and some of its extensions are presented

\subsection{Autoregressive Conditional Heteroskedasticity (ARCH) Family Model}

Every ARCH or GARCH family model requires two distinct specifications, namely: the mean and the variance equations (Atoi, 2014). The mean equation for a conditional heteroskedasticity in a return series, $y_{t}$ is given by

$$
y_{t}=E_{t-1}\left(y_{t}\right)+\varepsilon_{t}
$$

where

$$
\varepsilon_{t}=\phi_{t} \sigma_{t}
$$

The mean equation in equation (1) also applies to other GARCH family models. $E_{t-1}($.$) is the$ expected value conditional on information available at time $t-1$, while $\varepsilon_{t}$ is the error generated from the mean equation at time $\mathrm{t}$ and $\phi_{t}$ is the sequence of independent and identically distributed random variables with zero mean and unit variance.

The variance equation for an $\mathrm{ARCH}(\mathrm{p})$ model is given by

$$
\sigma_{t}^{2}=\omega+\alpha_{1} a_{t-1}^{2}+\ldots+\alpha_{p} a_{t-p}^{2}
$$


It can be seen in the equation that large values of the innovation of asset returns have bigger impact on the conditional variance because they are squared, which means that a large shock tends to follow another large shock and that is the same way the clusters of the volatility behave. So the $\mathrm{ARCH}(\mathrm{p})$ model becomes:

$$
a_{t}=\sigma_{t} \varepsilon_{t}, \quad \sigma_{t}^{2}=\omega+\alpha_{1} a_{t-1}^{2}+\ldots+\alpha_{p} a_{t-p}^{2}
$$

Where $\varepsilon_{t} \sim N(0,1)$ iid, $\omega>0$ and $\alpha_{i} \geq 0$ for $i>0$. In practice, $\varepsilon_{t}$ is assumed to follow the standard normal or a standardized student- $t$ distribution or a generalized error distribution (Tsay 2005).

\subsection{Asymmetric Power ARCH}

According to Rossi (2004), the asymmetric power ARCH model proposed by Ding,

Engel \& Granger (1993) given below forms the basis for deriving the GARCH family

models

Given that:

$$
\begin{gathered}
r=\mu+a_{t}, \\
\varepsilon_{t}=\sigma_{t} \varepsilon_{t}, \\
\varepsilon_{t} \sim N(0,1) \\
\sigma_{t}^{\delta}=\omega+\sum_{i=1}^{p} \alpha_{i}\left(\left|a_{t-i}\right|-\gamma_{i} a_{t-i}\right)^{\delta}+\sum_{j=1}^{q} \beta_{j} \sigma_{t-j}^{\delta},
\end{gathered}
$$

where 


$$
\begin{array}{ll}
\omega>0, & \delta \geq 0, \\
\alpha_{i} \geq 0 & i=1,2, \ldots, p \\
-1<\gamma_{i}<1 & i=1,2, \ldots, p \\
\beta_{j}>0 & j=1,2, \ldots, q
\end{array}
$$

This model imposes a Box-Cox transformation of the conditional standard deviation process and the asymmetric absolute residuals. The leverage effect is the asymmetric response of volatility to positive and negative "shocks".

\subsection{GARCH(p, q) Model:}

The mathematical model for the $\operatorname{GARCH}(p, q)$ model is obtained from equation (4) by letting $\delta=2$ and $\gamma_{i}=0, i=1, \ldots, p$ to be:

$$
a_{t}=\sigma_{t} \varepsilon_{t}, \quad \sigma_{t}^{2}=\omega+\sum_{i=1}^{p} \alpha_{i} a_{t-i}^{2}+\sum_{j=1}^{q} \beta_{j} \sigma_{t-j}^{2}
$$

Where $a_{t}=r_{t}-\mu_{t}\left(r_{t}\right.$ is the continuously compounded log return series), and

$\varepsilon_{t} \sim N(0,1)$ iid, the parameter $\alpha_{i}$ is the $\mathrm{ARCH}$ parameter and $\beta_{j}$ is the GARCH parameter, and $\omega>0, \alpha_{i} \geq 0, \beta_{j} \geq 0$, and $\sum_{i=1}^{\max (p, q)}\left(\alpha_{i}+\beta_{i}\right)<1$, (Rossi, 2004; Tsay, 2005 and Jiang, 2012). 
The restriction on $\mathrm{ARCH}$ and $\mathrm{GARCH}$ parameters $\left(\alpha_{i}, \beta_{j}\right)$ suggests that the volatility $\left(a_{i}\right)$ is finite and that the conditional standard deviation $\left(\sigma_{i}\right)$ increases. It can be observed that if $\mathrm{q}=0$, then the model GARCH parameter $\left(\beta_{j}\right)$ becomes extinct and what is left is an ARCH(p) model.

To expatiate on the properties of GARCH models, the following representation is necessary:

Let $\eta_{t}=a_{t}^{2}-\sigma_{t}^{2}$ so that $\sigma_{t}^{2}=a_{t}^{2}-\eta_{t}$. By substituting $\sigma_{t-i}^{2}=a_{t-i}^{2}-\eta_{t-i},(i=0, \ldots, q)$ into Eq. (4), the GARCH model can be rewritten as

$$
a_{t}=\alpha_{0}+\sum_{i=1}^{\max (p, q)}\left(\alpha_{i}+\beta_{i}\right) a_{t-i}^{2}+\eta_{t}-\sum_{j=1}^{q} \beta_{j} \eta_{t-j},
$$

It can be seen that $\left\{\eta_{t}\right\}$ is a martingale difference series (i.e., $E\left(\eta_{t}\right)=0$ and

$\operatorname{cov}\left(\eta_{t}, \eta_{t-j}\right)=0$, for $\left.j \geq 1\right)$. However, $\left\{\eta_{t}\right\}$ in general is not an iid sequence.

A GARCH model can be regarded as an application of the ARMA idea to the squared series $a_{t}^{2}$.

Using the unconditional mean of an ARMA model, results in this

$$
\mathrm{E}\left(a_{t}^{2}\right)=\frac{\alpha_{0}}{1-\sum_{i=1}^{\max (p, q)}\left(\alpha_{i}+\beta_{i}\right)}
$$

provided that the denominator of the prior fraction is positive. (Tsay, 2005)

When $\mathrm{p}=1$ and $\mathrm{q}=1$, we have $\operatorname{GARCH}(1,1)$ model given by:

$$
\begin{aligned}
& a_{t}=\sigma_{t} \varepsilon_{t}, \\
& \sigma_{t}^{2}=\omega+\alpha_{1} a_{t-1}^{2}+\beta_{1} \sigma_{t-1}^{2},
\end{aligned}
$$




\subsection{GJR-GARCH(p, q) Model}

The Glosten-Jagannathan-Runkle GARCH (GJRGARCH) model, which is a model that attempts to address volatility clustering in an innovation process, is obtained by letting $\delta=2$.

When $\delta=2$ and $0 \leq \gamma_{i}<1$,

$$
\begin{aligned}
& \sigma_{t}^{2}=\omega+\sum_{i=1}^{p} \alpha_{i}\left(\left|\varepsilon_{t-i}\right|-\gamma_{i} \varepsilon_{t-i}\right)^{2}+\sum_{j=1}^{q} \beta_{j} \sigma_{t-j}^{2} \\
& =\omega+\sum_{i=1}^{p} \alpha_{i}\left(\left|\varepsilon_{t-i}\right|^{2}+\gamma_{i}^{2} \varepsilon_{t-1}^{2}-2 \gamma_{i}\left|\varepsilon_{t-i}\right| \varepsilon_{t-i}\right)+\sum_{j=1}^{q} \beta_{j} \sigma_{t-j}^{2} \\
& \sigma_{t}^{2}= \begin{cases}\omega+\sum_{i=1}^{p} \alpha_{i}^{2}\left(1+\gamma_{i}\right)^{2} \varepsilon_{t-i}^{2}+\sum_{j=1}^{q} \beta_{j} \sigma_{t-j}^{2}, & \varepsilon_{t-i}<0 \\
\omega+\sum_{i=1}^{p} \alpha_{i}\left(1-\gamma_{i}\right)^{2} \varepsilon_{t-i}^{2}+\sum_{j=1}^{q} \beta_{j} \sigma_{t-j}^{2}, & \varepsilon_{t-i}>0\end{cases}
\end{aligned}
$$

i.e; $\quad \sigma_{t}^{2}=\omega+\sum_{i=1}^{p} \alpha_{i}\left(1-\gamma_{i}\right)^{2} \varepsilon_{t-i}^{2}+\sum_{i=1}^{p} \alpha_{i}\left\{\left(1+\gamma_{i}\right)^{2}-\left(1-\gamma_{i}\right)^{2}\right\} S_{i}^{-} \varepsilon_{t-i}^{2}+\sum_{j=1}^{q} \beta_{j} \sigma_{t-j}^{2}$

$$
\sigma_{t}^{2}=\omega+\sum_{i=1}^{p} \alpha_{i}\left(1-\gamma_{i}\right)^{2} \varepsilon_{t-1}^{2}+\sum_{j=1}^{q} \beta_{j} \sigma_{t-1}^{2}+\sum_{i=1}^{p} 4 \alpha_{i} \gamma_{i} S_{i}^{-} \varepsilon_{t-i}^{2}
$$

where

$$
S_{i}^{-}=\left\{\begin{array}{l}
1 \text { if } \varepsilon_{t-i}<0 \\
0 \text { if } \varepsilon_{t-i} \geq 0
\end{array},\right.
$$

Now define

$$
\alpha_{i}^{*}=\alpha_{i}\left(1-\gamma_{i}\right)^{2} \text { and } \gamma_{i}^{*}=4 \alpha_{i} \gamma_{i}
$$

then 


$$
\sigma_{t}^{2}=\omega+\sum_{i=1}^{p} \alpha_{i}\left(1-\gamma_{i}\right)^{2} \varepsilon_{t-i}^{2}+\sum_{j=1}^{q} \beta_{j} \sigma_{t-i}^{2}+\sum_{i=1}^{p} \gamma_{i}^{*} S_{i}^{-} \varepsilon_{t-1}^{2}
$$

Which is the GJRGARCH model (Rossi, 2004).

But when $-1 \leq \gamma_{i}<0$,

Then recall Eq. (8)

$$
\begin{gathered}
\sigma_{t}^{2}=\omega+\sum_{i=1}^{p} \alpha_{i}\left(\left|\varepsilon_{t-i}\right|-\gamma_{i} \varepsilon_{t-i}\right)^{2}+\sum_{j=1}^{q} \beta_{j} \sigma_{t-j}^{2} \\
=\omega+\sum_{i=1}^{p} \alpha_{i}\left(\left|\varepsilon_{t-i}\right|^{2}+\gamma_{i}^{2} \varepsilon_{t-1}^{2}-2 \gamma_{i}\left|\varepsilon_{t-i}\right| \varepsilon_{t-i}\right)+\sum_{j=1}^{q} \beta_{j} \sigma_{t-j}^{2} \\
\sigma_{t}^{2}=\left\{\begin{array}{lc}
\omega+\sum_{i=1}^{p} \alpha_{i}^{2}\left(1-\gamma_{i}\right)^{2} \varepsilon_{t-i}^{2}+\sum_{j=1}^{q} \beta_{j} \sigma_{t-j}^{2}, & \varepsilon_{t-i}>0 \\
\omega+\sum_{i=1}^{p} \alpha_{i}\left(1+\gamma_{i}\right)^{2} \varepsilon_{t-i}^{2}+\sum_{j=1}^{q} \beta_{j} \sigma_{t-j}^{2}, & \varepsilon_{t-i}<0
\end{array}\right. \\
\sigma_{t}^{2}=\omega+\sum_{i=1}^{p} \alpha_{i}\left(1+\gamma_{i}\right)^{2} \varepsilon_{t-i}^{2}+\sum_{j=1}^{q} \beta_{j} \sigma_{t-j}^{2}+\sum_{i=1}^{p} \alpha_{i}\left\{\left(1-\gamma_{i}\right)^{2}-\left(1+\gamma_{i}\right)^{2}\right\} S_{i}^{+} \varepsilon_{t-i}^{2} \\
=\omega+\sum_{i=1}^{p} \alpha_{i}\left(1+\gamma_{i}\right)^{2} \varepsilon_{t-i}^{2}+\sum_{j=1}^{q} \beta_{j} \sigma_{t-j}^{2}+\sum_{i=1}^{p} \alpha_{i}\left\{1+\gamma_{i}^{2}-2 \gamma_{i}-1-\gamma_{i}^{2}-2 \gamma_{i}\right\} S_{i}^{+} \varepsilon_{t-i}^{2}
\end{gathered}
$$

Where

$$
S_{i}^{+}=\left\{\begin{array}{l}
1 \text { if } \varepsilon_{t-i}>0 \\
0 \text { if } \varepsilon_{t-i} \leq 0
\end{array}\right.
$$

also define 


$$
\alpha_{i}^{*}=\alpha_{i}\left(1+\gamma_{i}\right)^{2} \text { and } \gamma_{i}^{*}=-4 \alpha_{i} \gamma_{i}
$$

then

$$
\sigma_{t}^{2}=\omega+\sum_{i=1}^{p} \alpha_{i}^{*} \varepsilon_{t-i}^{2}+\sum_{j=1}^{q} \beta_{j} \sigma_{t-i}^{2}+\sum_{i=1}^{p} \gamma_{i}^{*} S_{i}^{+} \varepsilon_{t-1}^{2}
$$

which allows positive shocks to have a stronger effect on volatility than negative shocks (Rossi, 2004). But when $p=q=1$, the GJRGARCH(1,1) model will be written as

$$
\sigma_{t}^{2}=\omega+\alpha \varepsilon_{t}^{2}+\gamma S_{i} \varepsilon_{t-1}^{2}+\beta \sigma_{t-1}^{2}
$$

\subsection{IGARCH(1, 1) Model}

The integrated GARCH (IGARCH) models are unit- root GARCH models. The IGARCH $(1,1)$ model is specified in Tsay (2005) and Grek (2014) as

$$
a_{t}=\sigma_{t} \varepsilon_{t} ; \quad \sigma_{t}^{2}=\alpha_{0}+\beta_{1} \sigma_{t-1}^{2}+\left(1-\beta_{1}\right) a_{t-1}^{2}
$$

Where $\varepsilon_{t} \sim \mathrm{N}(0,1)$ iid, and $0<\beta_{1}<1$, Ali (2013) used $\alpha_{i}$ to denote $1-\beta_{i}$.

The model is also an exponential smoothing model for the $\left\{a_{t}^{2}\right\}$ series. To see this, rewrite the model as

$$
\begin{aligned}
\sigma_{t}^{2} & =\left(1-\beta_{1}\right) a_{t-1}^{2}+\beta_{1} \sigma_{t-1}^{2} \\
& =\left(1-\beta_{1}\right) a_{t-1}^{2}+\beta_{1}\left[(1-\beta) a_{t-2}^{2}+\beta_{1} \sigma_{t-2}^{2}\right] \\
& =\left(1-\beta_{1}\right) a_{t-1}^{2}+\left(1-\beta_{1}\right) \beta_{1} a_{t-2}^{2}+\beta_{1}^{2} \sigma_{t-2}^{2} .
\end{aligned}
$$


By repeated substitutions, we have

$$
\sigma_{t}^{2}=\left(1-\beta_{1}\right)\left(a_{t-1}^{2}+\beta_{1} a_{t-2}^{2}+\beta_{1}^{2} a_{t-3}^{3}+\cdots\right),
$$

which is the well-known exponential smoothing formation with $\beta_{1}$ being the discounting factor (Tsay, 2005).

\subsection{TGARCH(p, q) Model}

The Threshold GARCH model is another model used to handle leverage effects, and a $\operatorname{TGARCH}(\mathrm{p}, \mathrm{q})$ model is given by the following:

$$
\sigma_{t}^{2}=\alpha_{0}+\sum_{i=1}^{p}\left(\alpha_{i}+\gamma_{i} N_{t=i}\right) a_{t-i}^{2}+\sum_{j=1}^{q} \beta_{j} \sigma_{t-j}^{2}
$$

where $N_{t-i}$ is an indicator for negative $a_{t-i}$, that is,

$$
N_{t-i}=\left\{\begin{array}{l}
1 \text { if } a_{t-i}<0 \\
0 \text { if } a_{t-i} \geq 0
\end{array}\right.
$$

and $\alpha_{i}, \gamma_{i}$, and $\beta_{j}$ are nonnegative parameters satisfying conditions similar to those of GARCH models, (Tsay, 2015). When $p=1, q=1$, the TGARCH(1, 1) model becomes:

$$
\sigma_{t}^{2}=\omega+\left(\alpha+\gamma N_{t-1}\right) a_{t-1}^{2}+\beta \sigma_{t-1}^{2}
$$

\subsection{NGARCH(p, q) Model}

The Nonlinear Generalized Autoregressive Conditional Heteroskedasticity (NGARCH) Model has been presented variously in literature by the following scholars: Hsieh \& Ritchken (2005), 
Lanne \& Saikkonen (2005), Malecka (2014) and Kononovicius \& Ruseckas (2015). The following model can be shown to represent all the presentations:

$$
h_{t}=\omega+\sum_{i=1}^{q} \alpha_{i} \varepsilon_{t-i}^{2}+\sum_{i=1}^{q} \gamma_{i} \varepsilon_{t-i}+\sum_{j=1}^{p} \beta_{j} h_{t-j}
$$

Where $h_{t}$ is the conditional variance, and $\omega, \beta$ and $\alpha$ satisfy $\omega>0, \beta \geq 0$ and $\alpha \geq 0$.

Which can also be written as

$$
\sigma_{t}=\omega+\sum_{i=1}^{q} \alpha_{i} \varepsilon_{t-i}^{2}+\sum_{i=1}^{q} \gamma_{i} \varepsilon_{t-i}+\sum_{j=1}^{p} \beta_{j} \sigma_{t-j}
$$

\subsection{SGARCH(p, q) Model}

The SGARCH model can be written as:

$$
\begin{aligned}
& Y_{t}=\eta_{t} \varepsilon_{t} \\
& \eta_{t}^{2}=\delta_{0}+\sum_{i=1}^{q} \delta_{i}\left(\eta_{t-i} \varepsilon_{t-i}\right)^{2}+\sum_{j=q+1}^{q+p} \delta_{j} \eta_{t+q-j}^{2},
\end{aligned}
$$

where $Y_{t}$ is the leading market return at time $t,\left\{\varepsilon_{t}\right\} \sim$ i.i.d. $N(0,1)$ is the innovation (or shock) of the market, and is hypothesized to be Gaussian. $\delta_{0}$ has to be positive and the remaining parameters nonnegative in order to ensure the positivity of $\eta_{t}^{2}$, (De Luca \& Loperfido, 2012)

\subsection{Simulation Procedure}

The simulation procedure here considers the following equations of $\operatorname{GARCH}(1,1)$ :

$$
\varepsilon_{t}=\sigma_{t} z_{t}
$$




$$
\sigma_{t}^{2}=a_{0}+\alpha_{1} \varepsilon_{t-1}^{2}+\beta_{1} \sigma_{t-1}^{2}
$$

The Case simulated is the case of financial time series where there are outliers at three level, namely: small values as 0.000005, 0.00006; medium values as 10, 50 and large values as 100, 500, at the following different time series length: 250, 500, 750, 1000, 1250 and 1500

The rugarch package of the R software was used to execute the simulation.

\subsection{Forecast Assessment}

The following are the criteria for Forecast assessments used:

1. Mean Absolute Error (MAE) has a formula $M A E_{j}=\frac{\sum_{i=1}^{n}\left|e_{i}\right|}{n}$. This criterion measures deviation from the series in absolute terms, and measures how much the forecast is biased. This measure is one of the most common ones used for analyzing the quality of different forecasts.

2. The Root Mean Square Error (RMSE) is given as $R M S E_{j}=\sqrt{\frac{\sum_{i}^{n}\left(\mathrm{y}_{i}-\mathrm{y}^{f}\right)^{2}}{n}}$ where $y_{i}$ is the time series data and $y^{f}$ is the forecast value of y (Caraiani, 2010).

For the two measures above, the smaller the value, the better the fit of the model (Cooray, 2008)

In this simulation study, $R M S E=\frac{\sum_{j}^{N} R M S E_{j}}{N}$ and $M A E=\frac{\sum_{j}^{N} M A E_{j}}{N}$ where $\mathrm{N}=1,000$, is the number of iterations or replications in the simulation study. Therefore, the model with the minimum RMSE and MAE result will be the preferred model 


\subsection{Results and Discussion}

\subsection{Results}

The results of the simulation carried out are presented in Table 1 to Table 8 below. 
Table 1: The RMSE and MAE values from the fGARCH family model at different levels of outlier of 0.000005, 0.00006 at different time series lengths

\begin{tabular}{|c|c|c|c|c|c|c|c|c|c|c|c|c|}
\hline Outlier & \multicolumn{12}{|c|}{$0.000005,0.00006$} \\
\hline Time series length $(\mathrm{T})$ & \multicolumn{2}{|c|}{250} & \multicolumn{2}{|c|}{500} & \multicolumn{2}{|c|}{750} & \multicolumn{2}{|c|}{1000} & \multicolumn{2}{|c|}{1250} & \multicolumn{2}{|c|}{1500} \\
\hline Model & RMSE & MAE & RMSE & MAE & RMSE & MAE & RMSE & MAE & RMSE & MAE & RMSE & MAE \\
\hline sGARCH & 4.931399 & 61.202956 & 7.021094 & 123.643002 & 8.618935 & 185.975292 & 9.945382 & 247.901383 & 11.15621 & 310.73484 & 12.21861 & 372.94698 \\
\hline gjrGARCH & 4.91953 & 61.17128 & 7.015205 & 123.499443 & 8.60339 & 185.62731 & 9.967985 & 248.371364 & 11.14907 & 310.66572 & 12.22246 & 372.89526 \\
\hline iGARCH & 4.890762 & 60.848528 & 7.03993 & 123.91656 & 8.57163 & 184.87379 & 9.971203 & 248.298866 & 11.1452 & 310.4949 & 12.20729 & 372.77983 \\
\hline TGARCH & 4.936581 & 61.365738 & 6.998018 & 123.271838 & 8.626176 & 186.112062 & 9.928296 & 247.502235 & 11.14536 & 310.59719 & 12.22121 & 372.87911 \\
\hline NGARCH & 4.90386 & 60.89776 & 7.007123 & 123.314485 & 8.598005 & 185.359426 & 9.977334 & 248.561108 & 11.11676 & 309.78808 & 12.21001 & 372.83915 \\
\hline
\end{tabular}

Table 2: The Ranks of The RMSE and MAE values from the fGARCH family model at different levels of outlier of $0.000005,0.00006$ at different time series lengths

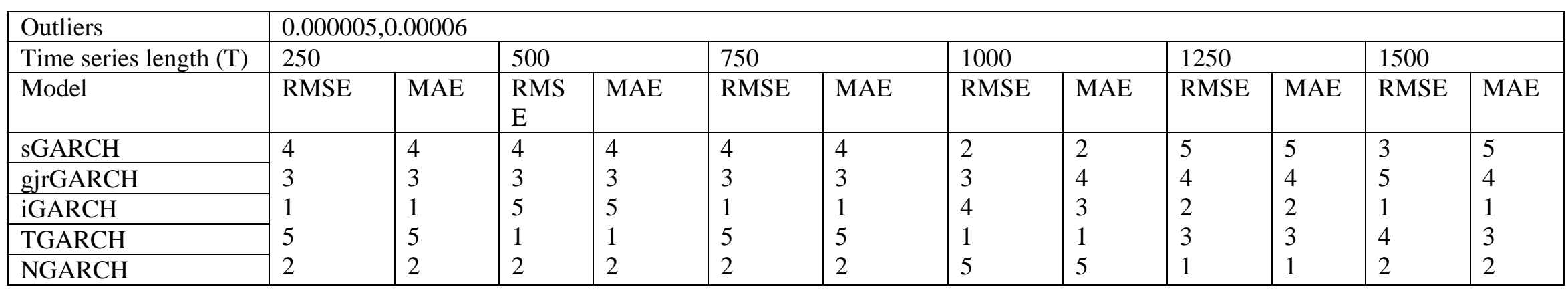


Table 3: The RMSE and MAE values from the fGARCH family model at different levels of outlier of 10, 50 at different time series lengths

\begin{tabular}{|c|c|c|c|c|c|c|c|c|c|c|c|c|}
\hline Outlier & 10,50 & & & & & & & & & & & \\
\hline $\begin{array}{l}\text { Time Series } \\
\text { Length }(\mathrm{T})\end{array}$ & \multicolumn{2}{|l|}{250} & \multicolumn{2}{|l|}{500} & \multicolumn{2}{|l|}{750} & \multicolumn{2}{|l|}{1000} & \multicolumn{2}{|l|}{1250} & \multicolumn{2}{|l|}{1500} \\
\hline Model & RMSE & MAE & RMSE & MAE & RMSE & MAE & RMSE & MAE & RMSE & MAE & RMSE & MAE \\
\hline sGARCH & 70.80231 & 118.59578 & 70.46127 & 118.58901 & 69.83468 & 118.25637 & 70.16223 & 119.31764 & 69.81693 & 119.30811 & 70.51907 & 121.11153 \\
\hline gjrGARCH & 71.69847 & 120.10907 & 70.6713 & 118.9951 & 69.96143 & 118.38869 & 68.57628 & 116.64949 & 67.85942 & 116.01714 & 69.43985 & 119.28377 \\
\hline iGARCH & 72.18174 & 123.33328 & 72.60727 & 124.69260 & 72.2639 & 123.0646 & 72.10861 & 122.67465 & 72.04126 & 123.14981 & 72.10363 & 123.82272 \\
\hline TGARCH & 71.9265 & 121.2603 & 72.07572 & 121.51990 & 72.07916 & 122.15585 & 71.73488 & 122.01710 & 72.03237 & 123.12899 & 72.10291 & 123.90638 \\
\hline NGARCH & 71.67376 & 120.60028 & 71.60875 & 120.43185 & 70.08789 & 118.50482 & 70.44886 & 119.71692 & 69.80573 & 119.24967 & 69.94543 & 120.09876 \\
\hline
\end{tabular}

Table 4: The Ranks of The RMSE and MAE values from the fGARCH family model at different levels of outlier of 10,50 at different time series lengths

\begin{tabular}{|c|c|c|c|c|c|c|c|c|c|c|c|c|}
\hline outliers & \multicolumn{12}{|l|}{10,50} \\
\hline $\begin{array}{l}\text { Time series } \\
\text { length }(\mathrm{T})\end{array}$ & \multicolumn{2}{|l|}{250} & \multicolumn{2}{|l|}{500} & \multicolumn{2}{|l|}{750} & \multicolumn{2}{|l|}{1000} & \multicolumn{2}{|l|}{1250} & \multicolumn{2}{|l|}{1500} \\
\hline Model & RMSE & MAE & RMSE & MAE & RMSE & MAE & RMSE & MAE & RMSE & MAE & RMSE & MAE \\
\hline sGARCH & 1 & 1 & 1 & 1 & 1 & 1 & 2 & 2 & 3 & 3 & 3 & 3 \\
\hline gjrGARCH & 3 & 2 & 2 & 2 & 2 & 2 & 1 & 1 & 1 & 1 & 1 & 1 \\
\hline iGARCH & 5 & 5 & 5 & 5 & 5 & 5 & 5 & 5 & 5 & 5 & 5 & 4 \\
\hline TGARCH & 4 & 4 & 4 & 4 & 4 & 4 & 4 & 4 & 4 & 4 & 4 & 5 \\
\hline NGARCH & 2 & 3 & 3 & 3 & 3 & 3 & 3 & 3 & 2 & 2 & 2 & 2 \\
\hline
\end{tabular}


Table 5: The RMSE and MAE values from the fGARCH family model at different levels of outlier of 100, 500 at different time series lengths

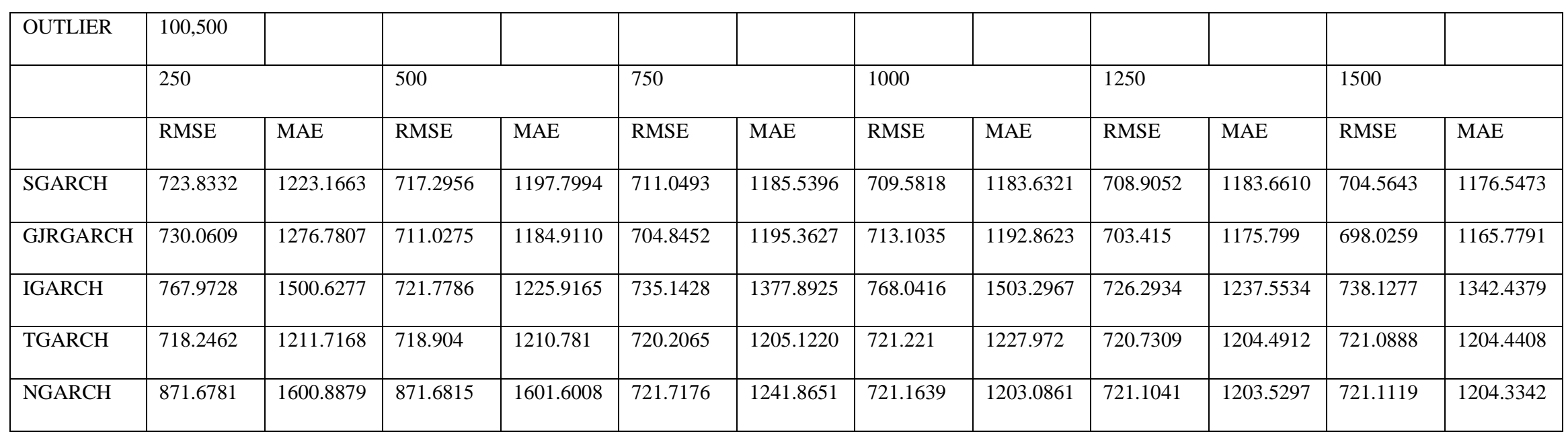

Table 6: The Ranks of The RMSE and MAE values from the fGARCH family model at different levels of outlier of 100, 500 at different time series lengths

\begin{tabular}{|c|c|c|c|c|c|c|c|c|c|c|c|c|}
\hline outliers & \multicolumn{12}{|c|}{100,500} \\
\hline $\begin{array}{l}\text { Time series } \\
\text { length (T) }\end{array}$ & \multicolumn{2}{|c|}{250} & \multicolumn{2}{|l|}{500} & \multicolumn{2}{|l|}{750} & \multicolumn{2}{|l|}{1000} & \multicolumn{2}{|l|}{1250} & \multicolumn{2}{|l|}{1500} \\
\hline Model & RMSE & MAE & RMSE & MAE & RMSE & MAE & RMSE & MAE & RMSE & MAE & RMSE & MAE \\
\hline sGARCH & 2 & 2 & 2 & 2 & 2 & 1 & 1 & 1 & 2 & 2 & 2 & 2 \\
\hline gjrGARCH & 3 & 3 & 1 & 1 & 1 & 2 & 2 & 2 & 1 & 1 & 1 & 1 \\
\hline iGARCH & 4 & 4 & 4 & 4 & 5 & 5 & 5 & 5 & 5 & 5 & 5 & 5 \\
\hline TGARCH & 1 & 1 & 3 & 3 & 3 & 3 & 4 & 4 & 3 & 4 & 3 & 4 \\
\hline NGARCH & 5 & 5 & 5 & 5 & 4 & 4 & 3 & 3 & 4 & 3 & 4 & 3 \\
\hline
\end{tabular}


Table 7: The Performances of the fGARCH family models at different levels of outliers and at different time series lengths using RMSE

\begin{tabular}{|l|c|c|c|c|c|c|}
\hline \multicolumn{7}{|c|}{ Forecast Statistics: RMSE } \\
\hline $\begin{array}{l}\text { Size of } \\
\text { Outlier }\end{array}$ & \multicolumn{5}{|c|}{ Time series length (T) } \\
\cline { 2 - 7 } & 250 & 500 & 750 & 1000 & 1250 & 1500 \\
\hline Small & iGARCH & TGARCH & iGARCH & TGARCH & NGARCH & iGARCH \\
\hline Medium & sGARCH & sGARCH & sGARCH & gjrGARCH & gjrGARCH & gjrGARCH \\
\hline Large & TGARCH & gjrGARCH & gjrGARCH & sGARCH & gjrGARCH & gjrGARCH \\
\hline
\end{tabular}

Table 8: The Performances of the fGARCH family models at different levels of outliers and at different time series lengths using MAE

\begin{tabular}{|l|c|c|c|c|c|c|}
\hline \multicolumn{7}{|c|}{ Torecast Statistic: MAE } \\
\hline Size of Outlier & \multicolumn{7}{|c|}{ Time series length (T) } \\
\cline { 2 - 7 } & 250 & 500 & 750 & 1000 & 1250 & 1500 \\
\hline Small & iGARCH & TGARCH & iGARCH & TGARCH & NGARCH & iGARCH \\
\hline Medium & sGARCH & sGARCH & sGARCH & gjrGARCH & gjrGARCH & gjrGARCH \\
\hline Large & TGARCH & gjrGARCH & sGARCH & sGARCH & gjrGARCH & gjrGARCH \\
\hline
\end{tabular}




\subsection{Discussion}

\subsubsection{GARCH models performance in the presence of outliers using the root mean square error (RMSE) from the results of the simulation}

When the additive outlier was small, iGARCH outperformed the other models at time series lengths (T) of 250, 750 and 1500, and TGARCH performed better than the other models at time series length (T) of 500 and 1000, while NGARCH performed better than the other models at time series length (T) of 1250 .

But for medium level of additive outliers, it can be clearly seen that the GARCH models that dominated were sGARCH and gjrGARCH. Whereas sGARCH performed better at time series lengths $(\mathrm{T})=250, \mathrm{~T}=500$ and $\mathrm{T}=750$, gjrGARCH outperformed the other models at $\mathrm{T}=1000$, $\mathrm{T}=1250$ and $\mathrm{T}=1500$.

Also for the large level of outliers, gjrGARCH dominated, performing better at time series lengths (T) of 500, 750, 1250 and 1500, while TGARCH performed better at time series length (T) of 250, and sGARCH outperformed the other models at $\mathrm{T}=1000$.

\subsubsection{GARCH models performance in the presence of outliers using the mean absolute error (MAE) from the results of the simulation}

For the small level of additive outliers, iGARCH dominated as it outperformed the other models at time series lengths $(\mathrm{T})$ of 250,750 and 1500 . TGARCH performed better than the other models at time series length (T) of 500 and 1000, while NGARCH outperformed the other models at time series length $(\mathrm{T})$ of 1250 . 
Coming to the medium level of additive outliers, it can also be seen that sGARCH and gjrGARCH dominated. While sGARCH performed better at time series lengths $(\mathrm{T})=250, \mathrm{~T}=$ 500 and $\mathrm{T}=750$, gjrGARCH on the other hand outperformed the other models at $\mathrm{T}=1000, \mathrm{~T}=$ 1250 and $\mathrm{T}=1500$.

Also for the large level of outliers, gjrGARCH dominated again, performing better at time series lengths (T) of 500, 1250 and 1500, sGARCH performed better than the other models at time series length (T) of 750 and 1000, while TGARCH outperformed the other models at $\mathrm{T}=250$.

\subsection{Summary}

This study has shown that different models performed better at different levels of outliers and at different time series lengths. This is in line with previous studies: Atoi (2014) modelling the volatility of stock returns using daily closing data of Nigerian Stock Exchange, found that GARCH $(1,1), \operatorname{PGARCH}(1,1,1)$ and $\operatorname{EGARCH}(1,1)$ with student's t distribution, and TGARCH with GED were the four best fitted models based on Schwarz Information Criterion, and the conclusions in Grek (2014), Chen, Min and Chen (2013), Dijk, Franses and Lucas (1999) and Demos (2000), that different models performed differently under different conditions; in this case, different levels of outliers.

\subsection{Conclusion}

Finding an optimal model for any time series, so that one can get good forecasting results with less prediction error, is one of the goals of analysis. To this effect, five different GARCH-Type models were simulated. The result of this study showed that with the presence of additive outliers, gjrGARCH dominated, especially for medium and large levels of outliers for large time 
series lengths, sGARCH dominated for lower time series lengths, irrespective of whether MAE or RMSE was used in the assessment.

\subsection{Recommendations}

The study therefore recommended that investors, financial analysts and researchers interested in stock prices and asset return should adapt gjrGARCH and sGARCH when outliers exist in their data.

\section{References}

Ali, G. (2013) "EGARCH, GJR-GARCH, TGARCH, AVGARCH, NGARCH, IGARCH, and APARCH Models for Pathogens at Marine Recreational Sites". Journal of Statistical and Econometric Methods, 2 (3): 57-73.

Atoi, N. V. (2014) "Testing volatility in Nigeria stock market using GARCH models". CBN Journal of Applied Statistics, 5: 65-93.

Bollerslev, T. (1986) “Generalized Autoregressive Conditional Heteroskedasticity”. Journal of Econometrics. 31: 307-327

Caraiani, P. (2010): Forecasting Romanian GDP using A BVAR model. Romanian Journal of Economic Forecasting. 4:76-87.

Chen, S., Min, W. \& Chen, R. (2013) "Model identification for Time Series with dependent innovations”. Statistica Sinica, 23: 873 - 899.

Cooray, T. M. J. A. (2008): Applied Time series Analysis and Forecasting. New Delhi:

Narosa Publishing House

De Luca, G., Genton, M. G. \& Loperfido, N. (2005) “A Multivariate Skew-GARCH Model”. Advances in Econometrics, 20: 33 -56

De Luca, G. \& Loperfido, N. (2012) "Modeling multivariate skewness in financial returns: a SGARCH approach". The European Journal of Finance, 1 -19 iFirst. http://dx.doi.org/10.1080/1351847X.2011.640342

Demos (2000) "Autocorrelation Function of Conditionally Heteroskedastic in Mean Models". Journal of Athens University of Economics and Business, Department of International and European Economic Studies, pp 1- 52. 
Dijk, D. V., Franses, P. H. \& Lucas, A. A. (1999) “Testing for ARCH in the Presence of Additive Outliers". Journal of Applied Econometrics, 14 (5) 539-562.

Ding, Z, Granger, C. W. J. \& Engle, R. F. (1993) “A long memory property of stock market returns and a new model”, Journal of Empirical Finance, 1, 83-106.

Duan, J. C., Gauthier, G., Simonato, J. G. \& Sasseville, C. (2006). “Approximating the GJR-GARCH and EGARCH option pricing models analytically”. Journal of Computational Finance, 9 (3) 1 -29.

Engle, R. F. (1982) “Autoregressive conditional heteroskedasticity with estimates of the Variance of United Kingdom inflation”. Econometrica, 50: 987-1007.

Glosten, L. R., Jagannathan, R. \& Runkle, D. E. (1993). “On the Relation between the Expected value and the volatility of the National excess return on stock". Journal of Finance, 48(5): 1779-1801

Grek, A. (2014) "Forecasting accuracy for ARCH models and GARCH(1,1) family which model does best capture the volatility of the Swedish stock market"? Statistics Advance Level Theses 15hp; Örebro University.

Hentschel, L. (1995) “All in the family Nesting symmetric and asymmetric GARCH models". Journal of Financial Economics, 39: 71-104

Higgins, M. L. \& Bera, A. (1992) “A class of Nonlinear ARCH Models”. International Economic Review, 33 (1): 137 -158

Hsieh, K. C. \& Ritchken, P. (2005) “An Empirical Comparison of GARCH Option Pricing Models". Review of Derivatives Research, 8 (3): 129 -150.

Jiang, W. (2012) "Using the GARCH model to analyse and predict the different stock markets" Master Thesis in Statistics, Department of Statistics, Uppsala University Sweden.

Kelkay, B. D. \& and G/Yohannes, E. (2014) “The Application of Garch Family Models to Some Agricultural Crop Products in Amhara National Regional State". Journal of Economics and Sustainable Development, 5: 24-35.

Kononovicius, A. \& Ruseckas, J. (2015) "Nonlinear GARCH Model and 1/f noise”. arXiv: 1412.6244v2[q-fin.ST].

Lanne, M. \& Saikkonen, P. (2005) "Nonlinear GARCH Models for Highly Persistent 
Volatility". Econometrics Journal, 8 (2): 251-276.

Malecka, M. (2014) “GARCH Class Models Performance in Context of High Market Volatility”. ACTA Universitatis Lodziensis Folia Oeconmica, 3: 253 -266

McQuarrie, A. D. \& Tsai, C. L. (2003) "Outlier Detection in Autoregressive Models" Journal of Computational and Graphical Statistics, 12 (2): 450-471.

Majose, R.V. (2010). "Volatility Models with Leverage Effect". A Doctoral Thesis. Universidad Carlos III De Madrid.

Nelson, D. (1991). "Conditional Heteroskedasticity in Asset Returns: A new approach", Econometrica, 59(2): 394-419.

Ragnarsson, F. J. (2011) "Comparison of Value-at-Risk Estimates from GARCH Models" A Master of Science Thesis, Department of Economics, Copenhagen Business School, November.

Rossi, E. (2004) "Lecture notes on GARCH models”. University of Pavia, March.

Sentana, E. (1995). "Quadratic ARCH Models”. The Review of Economic Studies. 62(4): $639-661$

Tsay, R. S. (2005) Analysis of financial time series, 2nd Edition. New Jersey: John Wiley \& Sons.

Taylor, S. (1986) Modelling Financial Time Series, Wiley, Chichster.

Wikipedia (2017). Autoregressive conditional heteroskedasticity (ARCH). https://en.wikipedia.org/wiki._(accessed 2017/07/10)

Zakoian, J. M. (1994) “Threshold heteroskedasticity models" Journal of Economic Dynamics and Control, 18(5): 931-955. 